\title{
Overestimation of Baseline Factor IX Activity in a Patient with Mild Hemophilia B by Soy Phosphatide-Containing aPTT Reagent in a One-Stage Assay
}

\author{
Michael Losos ${ }^{1}$, Janine Martin ${ }^{1}$, Paul Steele ${ }^{1}$, Cristina Tarango ${ }^{1}$, and Lori Luchtman-Jones ${ }^{1}$ \\ ${ }^{1}$ Cincinnati Children's Hospital Medical Center
}

June 4, 2020

\begin{abstract}
Testing and screening for factor deficiency using one-stage clotting assays is the most prevalent methodology, but it is susceptible to misestimating a patient's true coagulable state due to either the clot detection methodology or the aPTT reagents involved. In our case report, a 16-year-old with mild hemophilia B had a normal aPTT and factor IX activity using a new platform in the clinical laboratory. We evaluated the impact of instrument and aPTT reagents that differed in clot detection methodology, the activator and the source of phospholipid in one stage assays (OSA) for FIX:C and aPTT using plasma from this patient.
\end{abstract}

Abstract:

Testing and screening for factor deficiency using one-stage clotting assays is the most prevalent methodology, but it is susceptible to misestimating a patient's true coagulable state due to either the clot detection methodology or the aPTT reagents involved. In our case report, a 16-year-old with mild hemophilia B had a normal aPTT and factor IX activity using a new platform in the clinical laboratory. We evaluated the impact of instrument and aPTT reagents that differed in clot detection methodology, the activator and the source of phospholipid in one stage assays (OSA) for FIX:C and aPTT using plasma from this patient.

Introduction:

Laboratory testing for bleeding disorders includes two widely available screening assays for coagulation factor deficiencies, activated partial thromboplastin time (aPTT) and prothrombin time (PT). ${ }^{1-3}$ Clinicians must understand the potential limitations of screening and specific factor activity testing, as performed in their laboratory, to accurately reflect their patient's hemostatic status. The aPTT independently tests coagulation factors VIII, IX and XI, but also assesses contact factors XII, prekallikrein and high molecular weight kininogen. The aPTT shares screening of "common pathway" factors V, X, II and I with the PT, which independently screens factor VII. ${ }^{3}$ An isolated prolongation of the aPTT (with normal PT) would prompt the clinician to evaluate factors XI, IX and VIII for deficiency that might be associated with a bleeding phenotype. Normal PT and aPTT may provide reassurance against a severe deficiency of the screened coagulation factors. We report on a failure of the screening aPTT and a specific factor activity assay to detect mild factor IX deficiency.

A variation of the aPTT utilizing specific factor-deficient plasma can estimate the patient's factor VIII, IX or XI activity to detect deficiency or monitor coagulation factor infusions ${ }^{3-5}$ Variant aPTT testing can monitor unfractionated heparin effect or screen for a lupus anticoagulant. ${ }^{2}$ When screening or testing for hemophilia is desired, the aPTT should be free from heparin contamination and sensitive to low factor XI, IX or VIII activity, but have low sensitivity to factor XII deficiency or lupus anticoagulants. ${ }^{2}$ Most laboratories use a one-stage assay (OSA) for the aPTT. Patient plasma is mixed with an aPTT reagent, incubated briefly at 
$37^{\circ} \mathrm{C}$, and recalcified. Time to fibrin clot formation is measured. For specific factor assays, patient plasma is diluted with stock plasma deficient in the factor of interest, and the correction of the aPTT is compared to a standard curve of serial dilutions with known factor activity. ${ }^{1}$

Coagulation instruments differ in their methods for clot detection. Mechanical detection senses cessation of movement of a metal ball between two magnets. Photo-optical detection registers a change in optical density or turbidity. Various manufacturers offer aPTT reagents that differ in the contact activator (silica, ellagic acid, or kaolin) and phospholipid source (animal brain, plant-based or a combination). Clinicians must understand the strengths and pitfalls of their laboratory's platform (instrument + aPTT reagent), particularly when results deviate from expectations.

Case Description: A 16-year-old with mild hemophilia B (HB), baseline factor IX activity (FIX:C) 0.14-0.20 IU $\mathrm{mL}^{-1}$, had normal FIX:C and aPTT from annual comprehensive visit testing, despite no recent factor IX product infusion. Review of recent annual testing revealed FIX:C had been higher for the past few years, temporally corresponding with a change in the laboratory coagulation platform. The patient's de novo factor IX gene (F9) missense mutation in exon 8, c.1174 A>G (p.Asn392Asp), impacts subdomain 2 of the C-terminal catalytic protease domain ${ }^{6,7}$ and is consistent with type 2 HB.

Methods: Assent and consent had been given by the patient and his guardian, respectively, for participation in the institutional, IRB-approved tissue and data repository for bleeding disorders. Residual $3.2 \%$ sodium citrate buffered platelet-poor plasma (PPP) samples frozen at $-70^{\circ} \mathrm{C}$ were available for testing. The PPP was obtained from citrated whole blood samples via double centrifugation at $4440 \times \mathrm{g}$ for 3 minutes. Samples from several visits were assayed for FIX:C and aPTT on four coagulation instruments, using various aPTT reagents (Table 1 ).

Results:

The most disparate results of a normal range aPTT and increased FIX:C relative to historical values were generated using the clinical laboratory's platform which utilizes photo-optical detection of clot formation and an aPTT reagent with a purified soy-based phosphatide lipid. Other platforms generated prolonged aPTT and FIX:C levels in range for a diagnosis of mild hemophilia B (Table 2).

Discussion:

For the detection, classification and treatment of hemophilia, clinicians require laboratory tests with acceptable accuracy and precision. ${ }^{5}$ Historically the OSA aPTT should be sensitive to FIX:C of $<30 \mathrm{IU} \mathrm{mL}{ }^{-1} .{ }^{8}$ We demonstrate that coagulation test results are highly dependent upon the testing platform, which is a combination of the instrument's method of clot detection as well as the phospholipid source and activator in the aPTT reagent.

The OSA has been reported to misestimate activity in a subset of FVIII deficient patients/factor products, but this is less well-appreciated for other contact sequence factors, including factor IX. Over-estimation of FIX:C has been reported in $\mathrm{HB},{ }^{3}$ particularly type 2 HB. ${ }^{4}$ In physiologic coagulation, activated FIX interacts with coagulation cofactor VIIIa via its protease and epidermal-growth-factor-like 2 domains on the platelet surface. ${ }^{7}$ Our patient's protease domain mutation likely affects binding to the FVIII A2 subunit. ${ }^{6,7}$ Abnormal interaction of type $2 \mathrm{HB}$ plasma with soy phospholipids has been hypothesized based upon reduced sensitivity to FIX deficiency in type 2 HB plasmas tested with plant-based phosphatides. ${ }^{8,11,12}$

Our coagulation testing platforms varied in clot detection method, as well as both aPTT reagent phospholipid source and activator. Clot detection (mechanical vs. optical) seems of less impact than differences in fatty acid content and source of the aPTT reagent (Table 1). ${ }^{9,10}$ The historical concern about plant-based phosphatides is not widely known. ${ }^{11}$ In a three-way laboratory comparison of plasma FIX:C in 40 HB patients, Pouplard et al. $(2009)^{8}$ reported FIX:C overestimation in type 2, but not type 1, HB plasmas assayed with a soy phosphatide-containing aPTT reagent. An abnormal interaction of the mutated FIX with soy phospholipids was hypothesized. ${ }^{8}$ The effect of variation in contact activators is less clear, since phospholipid source and activators differ by reagent. Touri and Peerschke $(1986)^{12}$ examined three activators in (older) aPTT reagents 
and ranked kaolin less sensitive than ellagic acid or silica for deficiencies in factors VIII, IX and XII, although factor XI deficiency was equally detectable by all. Silica was more sensitive than ellagic acid to factor IX deficiency. ${ }^{12}$

Ultimately an aPTT reagent change was implemented.

Conclusion

For over 20 years, imperfect sensitivity has been described for some OSA platforms, not only for FVIII, but also for FIX and $\mathrm{FXI}^{8,11,12}$; however, clinicians may be less aware of potential differences for assays other than FVIII:C. ${ }^{11}$ Serious consideration should be given to selection of the coagulation platform for screening and factor activity testing. Differential sensitivities, at least in part attributed to the phospholipid source in aPTT reagents, could adversely affect identification and management of patients with hemophilia. While chromogenic assays do not suffer from this problem, they are not common as front-line testing.

We report a patient with known mild HB, attributable to rarer type $2 \mathrm{HB}$, who had a normal FIX:C and aPTT result in a recently-acquired coagulation platform employing photo-optical clot detection and a soy phospholipid aPTT reagent. Back-up testing on a different platform should be considered if OSA results are inconsistent or unexpected. More importantly, clinicians should work with their laboratories both on the choices for coagulation testing platform and in understanding the potential for over- or under-estimating results using OSAs.

Conflict of Interests

The authors claim no conflict of interests.

Bibliography

1. Favaloro EJ, Meijer P, Jemnings I, Sioufi J, Bonar RA, kitchen DP, Kershaw G, Lippi G. Problems and solutions in laboratory testing for hemophilia. Semin Thromb Hemost 2013;39:816-833.

2. Favaloro EJ, Kershaw G, Mohammed S, Lippi G. How to optimize activated partial thromboplastin time (APTT) testing: Solutions to establishing and verifying normal reference intervals and assessing APTT reagents for sensitivity to heparin, lupus anticoagulant and clotting factors. Semin Thromb Hemost 2019;45:22-35.

3. Winter EW, Flax SD, Harris NS. Coagulation testing in the core laboratory. Lab Medicine 2017;48:295313.

4. Wilmot HV and gray E. Potency estimates for recombinant factor IX in the one-stage clotting assay are influenced by more than just the choice of activated partial thromboplastin reagent. Haemophilia 2018;24:e363-368.

5. White GC II, Rosendaal F, Aledort LM, Lusher JM, Rothschild C, Ingerslev J; Factor VIII and Factor IX Subcommittee. Definitions in hemophilia. Recommendation of the scientific subcommittee on factor VIII and factor IX of the scientific and standardization committee of the International Society on Thrombosis and Haemostasis. Thromb Haemost 2001;85(3):560. PMID 11307831

6. Rallaplli PM, Kemball-Cook G, Tuddenham EG, Gomez K, Perkins SJ. An interactive mutation database for human coagulation factor IX provides novel insights into the phenotypes and genetics of hemophilia B. Thromb Haemost 2013;11:1329-40.

7. Schmidt AE, Bajaj SP. Structure-function relationships in factor IX and factor IXa. Trends Cardiovasc Med 2003;13:39-45.

8. Pouplard C, Trossaert M, Le Querrec A, Delahousse B, Giraudeau B, Gruel Y. Influence of source of phospholipids for APTT-based factor IX assays and potential consequences for the diagnosis of mild hemophilia B. Haemophilia 2009;15:365-368.

9. Mikaelsson M, Oswaldsson U, Sandberg H. Influence of phospholipids on the assessment of factor VIII activity. Haemophilia 1998;4:646-650.

10. Stevenson KJ, Easton AC, Curry A, Thomson JM, Poller L. The reliability of activated partial thromboplastin time methods and the relationship to lipid composition and ultrastructure. Thromb. Haemost 
$1986 ; 55: 250-8$.

11. Lawrie AS, Kitchen S, Purdy G, Mackie IJ, Preston FE, Machin SH. Assessment of Actin FS and Actin FSL sensitivity to specific clotting factor deficiencies. Clin Lab Haem 1998;20:179-186.

12. Turi DC, Peerschke EI. Sensitivity of three activated partial thromboplastin time reagents to coagulation factor deficiencies. Am J Clin Path 1986;85:43-49.

\section{Hosted file}

Table_1_May_20.docx available at https://authorea.com/users/329717/articles/456649overestimation-of-baseline-factor-ix-activity-in-a-patient-with-mild-hemophilia-b-bysoy-phosphatide-containing-aptt-reagent-in-a-one-stage-assay

\section{Hosted file}

Table_2_HTRS_FIX_abstract_Losos.docx available at https://authorea.com/users/329717/articles/ 456649-overestimation-of-baseline-factor-ix-activity-in-a-patient-with-mild-hemophiliab-by-soy-phosphatide-containing-aptt-reagent-in-a-one-stage-assay 